\title{
LA SEGURIDAD SOCIAL: ANÁLISIS DE SU PROBLEMÁTICA Y PROPUESTAS DE MEJORA
}

\author{
Juan Manuel Izar Landeta*, Carmen Berenice Ynzunza Cortés**, Roberto Sarmiento Rebeles***.
}

Izar-Landeta J.M., Ynzunza-Cortés C., Sarmiento-Rebeles $R$. La Seguridad Social: Análisis de su problemática y propuestas de mejora. Hitos de Ciencias Económico Administrativas 2012;18 (51):83-92.

\section{RESUMEN}

El número creciente de trabajadores a punto de retirarse presenta un problema serio para los sistemas de seguridad social en todo el mundo. Esto debido a que se estima que dichos programas se verán faltos de fondos en los próximos años.

Este ensayo comenta algunas de las variables más importantes relacionadas con esta temática. Estas incluyen la edad de retiro, el manejo financiero de las cuentas de retiro y esquemas flexibles de jubilación, entre otros.

También se incluyen algunas recomendaciones prácticas para solventar este problema.
Izar-Landeta J.M., Ynzunza-Cortés C., Sarmiento-Rebeles R. Social Security: Analysis of its problems and improvement proposals. Hitos de Ciencias Económico Administrativas 2012;18 (51):83-92.

\section{ABSTRACT}

The increasing number of workers approaching retirement age poses a challenge to social security programs all around the world. This is because these programs are projected to get underfunded in the coming years.

This essay comments some of the most important variables involved in such an important topic. These include retirement age, financial management of retirement accounts and flexible retirement schemes, amongst others.

Several practical recommendations to solve this problem are included.

Key words: Demographic change. Retirement benefits. Social security programs. Retirement age. Flexible retirement schemes. Individual Retirement Account.
Palabras clave: Cambio demográfico. Beneficios de retiro. Programas de seguridad social. Edad de retiro. Esquemas de jubilación flexibles. Cuenta individual de retiro.

DIRECCIÓN PARA RECIBIR CORRESPONDENCIA: Valle de Caldera 111, Frac. Miravalle, San Luis Potosí, S. L. P., C. P. 78214 Correo electrónico: jmizar@uaslp.mx

ste ensayo comenta los principales problemas que han llevado a los sistemas de seguridad social a una situación crítica en casi todas las naciones, lo que sin duda es tema de suma importancia por todo lo que implica y que obliga a buscar soluciones de fondo.
La causa principal de esta crisis es el cambio demográfico, ya que los esquemas de reparto o beneficios definidos (BD) que se pusieron en boga a mediados del siglo pasado al término de la Segunda Guerra Mundial, ya no son viables financieramente, puesto que no hay presupuesto que alcance para pagar los salarios de retiro de un número creciente de jubilados. Dichos esquemas fueron ideados para las

\footnotetext{
* Doctor en Administración. Facultad de Ingeniería. Universidad Autónoma de San Luis Potosí.

** Universidad Tecnológica de Querétaro.

*** Facultad de Ingeniería. Universidad Autónoma de San Luis Potosí.
}

Fecha de recibido: 9 de febrero de 2012 Fecha de aceptación: 28 marzo de 2012. 
condiciones de aquella época, las cuales han cambiado drásticamente, poniendo en jaque su subsistencia, la que se complica por las siguientes causas (Izar, 2010):

- Hay un número creciente de jubilados con la llegada de los baby boomers ${ }^{1}$ al retiro, ya que dicha generación es más numerosa que la que le sigue, que es la generación $\mathrm{X}$ y para los sistemas $\mathrm{BD}$, que fondean los pagos jubilatorios con los impuestos de los trabajadores activos, su sustentabilidad ya no es posible.

- Con los avances de la medicina la esperanza de vida de las personas ha aumentado, lo que implica mayores periodos de pago de los beneficios de retiro a los jubilados. Amediados del siglo pasado la esperanza de vida de la población en México se ubicaba en 56 años, ahora dicho valor oscila entre 76 y 80 años, lo que significa pagar los salarios de retiro durante 20 años adicionales al menos.

- La mayoría de los sistemas BD no incluyen el ahorro para el retiro, el cual es un elemento indispensable para lograr su solvencia financiera.

- Tales esquemas (BD) establecen las tasas de reemplazo ${ }^{2}$ con base en la antigüedad en el empleo y no en la edad del individuo, lo que ha traído como consecuencia que muchos trabajadores jóvenes se jubilen.

- Muchos sistemas BD definen el salario de retiro con base en el último salario del empleado y no en los salarios promedio durante su vida activa, actualizados por el índice de inflación.

- Muchos sistemas no tienen salarios reguladores con topes máximos.

Para darse una idea de la magnitud de tales cambios, Kinsella y He (2009) afirman que el envejecimiento de la población es el proceso demográfico más importante en el tercer milenio, el cual ha comenzado recientemente en los países menos desarrollados. Presentan un estadístico de los 25 países con mayores porcentajes de personas de 65 años o más de edad, que no incluye a Estados Unidos (Ver Tabla I).

\footnotetext{
${ }^{1}$ La generación de los baby boomers se conforma con todos los nacidos después de la Segunda Guerra Mundial, en el periodo de 1946 a 1964. En Estados Unidos se compone de 76 millones de personas, mientras que la generación siguiente, la $\mathrm{X}$, la forman sólo 51 millones, lo que hace un déficit intergeneracional de 25 millones de personas.

${ }^{2}$ La tasa de reemplazo es el cociente del salario de retiro y el último devengado como trabajador activo.
}

Es evidente que los países europeos son los que tienen mayores porcentajes de viejos. En cuanto a la participación de los viejos en la fuerza laboral de las naciones, de un reporte del LABORSTA (2011) -departamento estadístico de la Organización Internacional del Trabajo-, en la tabla II se presentan datos de 5 países de la Organización para la Cooperación y el Desarrollo Económico (OCDE) de 1995 a la fecha y su proyección al año 2015, sobre la participación de personas en edades comprendidas entre 55 y 64 años, separadas por género (Ver Tabla II).

Por su parte la Tabla III contiene la misma información pero para personas mayores de 65 años.

Se prevé que las personas comprendidas en ambas categorías tendrán mayor participación en la fuerza laboral en los años venideros, después de haber sido lo contrario en la década de los 90 como consecuencia

Tabla I. Los 25 países más viejos al año 2008.

\begin{tabular}{cc}
\hline País & $\begin{array}{c}\text { \% de población de 65 } \\
\text { años o más de edad }\end{array}$ \\
\hline Japón & 21.6 \\
Italia & 20.0 \\
Alemania & 20.0 \\
Grecia & 19.1 \\
Suecia & 18.3 \\
España & 17.9 \\
Austria & 17.7 \\
Bulgaria & 17.6 \\
Estonia & 17.6 \\
Bélgica & 17.5 \\
Portugal & 17.4 \\
Croacia & 17.0 \\
Latvia & 16.9 \\
Georgia & 16.6 \\
Finlandia & 16.6 \\
Francia & 16.3 \\
Eslovenia & 16.3 \\
Ucrania & 16.1 \\
Inglaterra & 16.0 \\
Suiza & 16.0 \\
Lituania & 16.0 \\
Dinamarca & 15.7 \\
Hungría & 15.6 \\
Serbia & 15.2 \\
Noruega & 15.0 \\
\hline
\end{tabular}

Fuente: Kinsella y He (2009). 
Tabla II. Datos de 5 países miembros de la OCDE en edades entre 55 y 64 años.

\begin{tabular}{|c|c|c|c|c|c|c|c|c|c|c|}
\hline \multirow[b]{3}{*}{ Año } & \multicolumn{10}{|c|}{ País } \\
\hline & \multicolumn{2}{|c|}{ Canadá } & \multicolumn{2}{|c|}{ Alemania } & \multicolumn{2}{|c|}{ Holanda } & \multicolumn{2}{|c|}{ Inglaterra } & \multicolumn{2}{|c|}{$\begin{array}{c}\text { Estados } \\
\text { Unidos }\end{array}$} \\
\hline & $\mathrm{H}$ & M & $\mathbf{H}$ & $M$ & $\mathrm{H}$ & $M$ & $\mathbf{H}$ & $\mathbf{M}$ & H & M \\
\hline 1995 & 58.3 & 36.2 & 54.3 & 31.5 & 41.4 & 18.5 & 62.4 & 40.7 & 66.0 & 49.2 \\
\hline 2000 & 60.7 & 41.4 & 51.3 & 32.4 & 50.7 & 26.3 & 63.3 & 42.6 & 67.3 & 51.9 \\
\hline 2005 & 66.8 & 49.3 & 61.9 & 43.8 & 59.5 & 36.4 & 67.8 & 48.8 & 69.3 & 57.1 \\
\hline 2010 & 68.4 & 56.7 & 70.6 & 54.2 & 67.5 & 44.6 & 69.2 & 50.5 & 70.0 & 60.2 \\
\hline 2015 & 71.0 & 60.7 & 71.4 & 55.6 & 71.1 & 49.1 & 72.1 & 56.3 & 71.4 & 63.2 \\
\hline
\end{tabular}

Fuente: LABORSTA Internet (2011).

Tabla III. Datos de 5 países miembros de la OCDE en edades de 65 años o más.

\begin{tabular}{|c|c|c|c|c|c|c|c|c|c|c|}
\hline \multirow[b]{3}{*}{ Año } & \multicolumn{10}{|c|}{ País } \\
\hline & \multicolumn{2}{|c|}{ Canadá } & \multicolumn{2}{|c|}{ Alemania } & \multicolumn{2}{|c|}{ Holanda } & \multicolumn{2}{|c|}{ Inglaterra } & \multicolumn{2}{|c|}{$\begin{array}{l}\text { Estados } \\
\text { Unidos }\end{array}$} \\
\hline & H & $\mathbf{M}$ & $\mathrm{H}$ & $\mathbf{M}$ & $\mathbf{H}$ & M & $\mathbf{H}$ & $\mathbf{M}$ & $\mathbf{H}$ & M \\
\hline 1995 & 9.9 & 3.4 & 4.2 & 1.6 & 5.6 & 1.0 & 8.2 & 3.2 & 16.8 & 8.8 \\
\hline 2000 & 9.4 & 3.3 & 4.4 & 1.5 & 4.3 & 1.4 & 7.8 & 3.4 & 17.7 & 9.4 \\
\hline 2005 & 12.1 & 4.9 & 5.1 & 2.2 & 7.8 & 2.1 & 9.0 & 4.4 & 19.8 & 11.5 \\
\hline 2010 & 16.2 & 7.5 & 5.7 & 2.8 & 9.4 & 3.2 & 11.3 & 6.4 & 22.1 & 13.8 \\
\hline 2015 & 17.1 & 8.2 & 5.9 & 2.9 & 10.9 & 4.0 & 11.7 & 6.4 & 23.8 & 15.9 \\
\hline
\end{tabular}

de los programas de jubilación anticipada, lo cual es confirmado por Cutler en un estudio reciente (2011).

Con datos del Health and Retirement Study (HRS) del periodo de 1992 a 2008, Cahill y colaboradores (2011) han encontrado que el $15 \%$ de los americanos viejos con empleos de carrera, volvieron a la fuerza laboral después de haberse jubilado y la probabilidad de haber vuelto a trabajar aumentaba si las personas eran más jóvenes, con mejor estado de salud, o si contaban con un plan de aportaciones definidas (CD). Las mujeres es más probable que vuelvan al empleo si tienen niños dependientes en casa.

Por lo que respecta a la esperanza de vida, la Tabla IV muestra los valores de esta variable en México para hombres y mujeres de los años 1930 al 2005, así como los valores proyectados para los años 2030 y 2050 (Consejo Nacional de Población, 2005).
Es notorio que los mexicanos viven ahora más años y el valor de la esperanza de vida tenderá a aumentar en el futuro, por los avances en el campo de la medicina.

Tabla IV. Esperanzas de vida al nacer en México por sexo.

\begin{tabular}{ccc}
\hline & \multicolumn{2}{c}{ Sexo } \\
\cline { 2 - 3 } Año & Hombres & Mujeres \\
\hline 1930 & 35.0 & 37.4 \\
2000 & 71.5 & 76.5 \\
2005 & 72.2 & 77.0 \\
2030 & 76.6 & 81.0 \\
2050 & 79.9 & 83.9 \\
\hline
\end{tabular}

Fuente: LABORSTA Internet (2011). 
En tales circunstancias, han cobrado auge los sistemas de cuentas individuales o aportaciones definidas denominados CD-, los cuales consisten en cuentas de ahorro para el retiro, a las cuales hacen aportaciones los trabajadores, las empresas e incluso el gobierno, de modo que lo que se acumule durante la vida activa del empleado, junto con los intereses que se generen por su buen manejo financiero, será lo que subsidie los pagos de jubilación de los trabajadores, de modo que los beneficios de retiro dependerán de lo que se acumule en la cuenta durante la vida activa del trabajador.

Como puede verse, la solución no es fácil y requiere la participación de muchos actores sociales, incluyendo por supuesto a los gobiernos, quienes deben asumir un papel regulador y aportador que garantice un retiro digno a sus jubilados.

Al respecto Hayashi y colaboradores (2010) señalan que para maximizar las oportunidades que trae consigo el envejecimiento de la sociedad hay 3 factores clave: 1) Colaboración efectiva entre los actores clave, como son las instituciones financieras, los proveedores del cuidado de la salud, el gobierno y los ciudadanos; 2) Cambio de paradigmas, hacia nuevas estructuras que incentiven la planeación a largo plazo y valoren la ancianidad como una etapa productiva de la vida; y 3 ) integrar soluciones de jubilación con el cuidado de la salud.

Algunas organizaciones internacionales como el Banco Mundial (BM) y la OCDE, preocupados por estas circunstancias, han emitido algunos lineamientos al respecto.

Entre las recomendaciones del Banco Mundial se cuentan las siguientes: Reducir los beneficios de retiro, elevar la edad de retiro, incrementar las aportaciones a las cuentas de retiro, aumentar los presupuestos de seguridad social y disminuir las inequidades existentes respecto a los beneficios de retiro (Holzmann, 2000). Para esto el Banco sugiere implementar sistemas de pilares múltiples, uno con fondeo obligatorio público y privado y otro con fondeo privado voluntario.

PorsupartelaOCDEhaemitidoalgunarecomendaciones, tales como: Eliminar los incentivos financieros de la jubilación anticipada y los desincentivos de la jubilación tardía, incrementar las oportunidades de empleo para las personas maduras, disminuir los beneficios de jubilación e incrementar las aportaciones a las cuentas de retiro, proveer los ingresos de retiro con impuestos, transferencias y ahorros y manejar las cuentas de manera diversificada a fin de disminuir el riesgo, cuidar los costos del cuidado de la salud de las personas de la tercera edad, fortalecer la infraestructura de los mercados financieros, establecer una estructura regulatoria efectiva y difundir estas políticas entre la población, con la finalidad de alcanzar la comprensión y el apoyo público (Visco, 1999).

En nuestro país las dos instituciones de seguridad social más importantes son el Instituto Mexicano del Seguro Social (IMSS) y el Instituto de Seguridad y Servicios Sociales de los Trabajadores del Estado (ISSSTE), quienes proporcionan este servicio a 60 millones de derechohabientes, de los cuales el IMSS absorbe al $80 \%$ y el ISSSTE el $20 \%$ restante. Ambas instituciones han modificado sus leyes anteriores, buscando con las reformas su mejora operativa y la viabilidad financiera de sus sistemas pensionarios.

\section{Revisión de la literatura}

Hay muchas variables implicadas en esta problemática de la seguridad social que inciden en su solvencia financiera, las cuales se comentan enseguida.

\section{Edad de retiro}

Establecer una edad de retiro ha sido un tema polémico entre políticos, académicos y ejecutivos de las empresas.

La edad de retiro es una variable que incide directamente en la solvencia financiera de la seguridad social, ya que si un empleado se jubila a una edad mayor, durará más tiempo haciendo aportaciones a su cuenta individual de retiro y con ello acumulará una cantidad de recursos mayor para su jubilación y por otra parte, gozará de los beneficios de retiro durante menos tiempo, lo que constituye un alivio a la presión financiera de los sistemas jubilatorios.

La seguridad social norteamericana buscando que los trabajadores maduros dejasen sus puestos laborales para los empleados jóvenes, estableció la jubilación anticipada a partir de los 62 años, con la condicionante que quienes la toman reciben menores beneficios de retiro, dependiendo de su edad normal de retiro. 
Sin embargo, con el cambio demográfico que se aproxima, se están tomando medidas en el sentido opuesto, buscando motivar a los trabajadores viejos a permanecer más años en sus puestos de trabajo.

Hay contradicciones en cuanto al rendimiento laboral de los trabajadores más viejos, pues algunos sostienen que los empleados maduros tienen más experiencia, mientras que otros señalan que los empleados viejos tienen menos aptitudes físicas e intelectuales para desarrollar sus labores de manera eficiente, lo cual podría ser válido en el caso de labores con altas demandas físicas.

Varios estudiosos consideran que fijar una edad obligatoria de jubilación es una medida discriminatoria (Nelson, 2002; Sargeant, 2004; Davies, 2005).

Otros investigadores han efectuado estudios para determinar los factores que influyen en la decisión de retiro de las personas, algunos afirman que el hecho de establecer impuestos a los trabajadores viejos, les induce a tomar la jubilación anticipada (Cremer et al., 2004; Duval, 2004).

La mayoría de los estudiosos coinciden que un mal estado de salud lleva a los trabajadores a jubilarse antes (Hall y Johnson, 1980; Burtless, 1986; Anderson et al., 1986; Dwyer y Mitchell, 1999).

Asimismo la mayoría de los autores afirman que con más bienes y una mejor situación económica, los empleados tienden a jubilarse antes (Hall y Johnson, 1980; Fields y Mitchell, 1984; Burtless, 1986; Anderson et al., 1986; Coile y Gruber, 2007).

En cuanto al efecto del desempleo en la decisión de retiro, hay posiciones encontradas, ya que Anderson y colaboradores (1986) señalan que si las oportunidades de empleo bajan, las personas tienden a jubilarse después, mientras que Bould (1980) dice lo contrario. Filer y Petri (1988) concluyen que los empleados con trabajos estresantes y repetitivos se jubilan antes que los que desempeñan puestos de trabajo de mayor perfil profesional.
Por su parte Cahill y colaboradores (2006) afirman que los americanos prefieren retirarse gradualmente y no de manera total.

Williamson y McNamara (2003) comentan que no sólo los aspectos económicos influyen en la decisión de retiro del empleado, sino también su trayectoria de vida. Por su parte Cerezo y Topa (2008) encuentran que el control personal es importante en el proceso de prejubilación y en su calidad de vida como jubilados. Como puede verse, el tema de la edad de retiro es controversial, pues hay quienes consideran que no debe aumentarse, ya que es un derecho de los empleados, mientras que otros piensan que de no hacerse, sería muy complicado garantizar la sustentabilidad financiera de la seguridad social.

\section{Condiciones financieras de la jubilación}

Puesto que ahora la solvencia financiera de la seguridad social está bajo la lupa, resulta imprescindible ahorrar para el retiro durante la vida activa del trabajador, lo cual ha traído consigo el auge de los sistemas CD, que cada vez ganan mayor popularidad, en sustitución de los anteriores esquemas BD.

Una variable muy importante en los sistemas de cuentas individuales es su rendimiento financiero (Levine, 2005; Izar, 2006), por lo cual los fondos deben invertirse buscando maximizar el rendimiento financiero para el riesgo manejado.

En varios países latinoamericanos, incluyendo por supuesto a México, las administradoras de los fondos de retiro ofrecen rendimientos muy bajos, ya que cobran elevadas comisiones, lo cual somete a serio cuestionamiento su proceder.

En un estudio reciente, Christman (2010) describe las etapas que pueden tomar los empleadores para ayudar a sus empleados a una mejor transición a la jubilación $y$, a su vez, maximizar el retorno sobre la inversión en su plan de retiro, para fondear sus ahorros. Dichas etapas incluyen calcular las tasas de reemplazo, tanto individuales y del plan, así como traer profesionales financieros que asesoren a los empleados.

En cuanto a fondos de jubilación de otras instituciones, como el caso de las universidades públicas en México, la SEP les condiciona que las aportaciones que han 
obtenido del gobierno federal para incrementar sus fondos se depositen en fideicomisos específicos, sujetos a un manejo transparente.

En Estados Unidos, Vora y McGinnis (2000) recomiendan a los jubilados invertir sus fondos de retiro en acciones y no en bonos, ya que producen mayores rendimientos financieros.

Franklin (2011) sugiere un proceso de 5 etapas para alcanzar una jubilación segura: 1) Hacer un chequeo del ahorro necesario para el retiro, 2) asegurarse de obtener lo máximo de su plan de retiro, 3) trabajar durante más tiempo, 4) convertir los ahorros de retiro en una mensualidad adecuada que no se vaya a agotar y 5) demorar la recepción de los beneficios de seguridad social.

\section{La jubilación flexible, una opción atractiva}

Muchos investigadores hablan que uno de los problemas de las organizaciones en esta era del conocimiento es precisamente la de administrar su conocimiento, aspecto que se ha convertido en tema obligado de estudio para académicos e instituciones, ya que con el inminente retiro de un gran número de trabajadores que están alcanzando la edad de jubilación, las organizaciones que no tomen medidas al respecto, se enfrentarán a una «fuga de inteligencias profundas», que consiste en los conocimientos tácitos y no explícitos de los boomers, que sólo los brinda la experiencia (Aiman-Smith et al., 2006).

Para darse una idea de lo que esto representa en Estados Unidos, Dychtwald y Baxter (2007) afirman que a medida que la generación de los boomers se jubile, las organizaciones enfrentarán una fuga masiva de cerebros, cuyo impacto ya comienzan a sentir. Algunas instituciones se están dando cuenta de la imperiosa necesidad de cambiar sus políticas laborales para adaptarse a las nuevas realidades demográficas. Erickson (2008) señala que los puestos de trabajo requerirán de los empleados viejos para llenar la brecha de talento entre la oferta y la demanda de empleos. El crecimiento de los empleos basados en el conocimiento aliviará la demanda de trabajo físico y reforzará la aceptación de los trabajadores maduros. Con la escasez creciente de talento y los avances de la tecnología de la información, será posible trabajar en cualquier lugar a cualquier hora.
De un estudio reciente, Byham (2008) comenta que una de las estrategias de las organizaciones norteamericanas es retener a sus empleados viejos más allá de su edad normal de jubilación, preferible a tener que recontratarlos, lo cual aplica especialmente en el caso de puestos gerenciales y administrativos.

Geber (2000) habla de la interesante iniciativa de Monsanto, quien en 1991 implantó un innovador programa denominado «Retiree Resource Corps», al cual acuden los trabajadores jubilados o los que están próximos al retiro. Cuando la gente entra al programa, especifica qué clase de trabajo desearía llevar a cabo. Algunas veces lo toman quienes aspiran a participar sólo en proyectos específicos, otras veces los que quieren puestos con menos horas laborales o menos estrés. Los jubilados se asignan a los puestos dependiendo de las necesidades de la compañía. La medida ha generado importantes ahorros a la corporación.

Ante tales circunstancias, la jubilación flexible luce como una opción atractiva, ya que permite a los trabajadores permanecer en sus puestos de trabajo, lo que trae consigo dos importantes beneficios a las organizaciones:

1. Al permanecer como trabajadores activos, no se hacen pagos de pensiones a estos empleados, lo cual ayuda a la solvencia financiera de sus planes de retiro.

2. Se retiene el conocimiento de estos empleados dentro de las instituciones.

La jubilación flexible puede adoptar alguna de las siguientes opciones (Izar et al., 2011):

- Tipo de trabajo. Poner al alcance de los empleados la posibilidad de cambiar sus actividades o tipo de trabajo, ya que a mayores edades los trabajos físicamente demandantes o con altas cargas de estrés, podrían cambiarse por otros menos pesados.

- Horario de trabajo. Al dar flexibilidad de horario, muchos trabajadores dispondrían de tiempo libre para dedicarlo a otras cosas.

- Lugar de trabajo. En esta época, con los avances de la tecnología de la información, muchos trabajos pueden hacerse desde casa. 
- Trabajo parcial. Esto es parte de lo que denominan «trabajos puente» o «retiro parcial», que son cada vez más frecuentes y consisten en hacer posible a los empleados la disminución de sus horas laborales y recibir un pago proporcional al tiempo trabajado.

Otros autores hablan de la jubilación en fases, como Noble y Harper (2010) quienes afirman que son una solución a varias realidades sociales: la recesión económica global, la incertidumbre en los costos del cuidado de la salud, los potenciales efectos sobre la fuerza laboral de una generación de baby boomers que envejece y el aumento en su expectativa de vida. Para que un programa de este tipo tenga éxito, deberá alinearse con el programa de beneficios globales de la organización mediante la modificación de su normativa.

\section{El papel que debe asumir el Estado}

Todos los gobiernos de las naciones tienen como objetivo garantizar a sus ciudadanos el bienestar, para lo cual les proveen diversos servicios, tales como educación, vivienda, salud, empleo y por supuesto, la seguridad social.

El objetivo de la seguridad social es lograr el bienestar de los trabajadores que llegan a la jubilación, de modo que tengan a su alcance beneficios dignos de retiro y servicios de atención y cuidado de la salud.

Para lograr esto, el Estado debe asumir un papel preponderante como regulador de la seguridad social y aportador, distribuyendo parte de los ingresos que recauda a las cuentas de retiro de los trabajadores.

Desde el nacimiento de la seguridad social a finales del siglo XIX, cuando el canciller alemán Otto von Bismarck vislumbró la necesidad de proveer este servicio a los ciudadanos, se implementaron los seguros de enfermedades, accidentes e invalidez y vejez.

Hay incluso autores que afirman que la seguridad social es parte fundamental en la construcción de la democracia de las naciones (Cortés, 2006).

En algunos países como México, el Estado ha adoptado el modelo neoliberal, donde el mercado rige la asignación de los recursos, lo que no debe aplicar para el caso de la educación pública ni la seguridad social, pues estos dos importantes pilares para el desarrollo de cualquier país, no deben dejarse a las libres fuerzas del mercado. Desgraciadamente el Estado ha caminado en la dirección opuesta y en el caso de la seguridad social, ha tratado de hacer un gran negocio con el manejo de los fondos pensionarios, por lo que hace falta un modelo más funcional (Campos et al., 2001).

El Estado debe garantizar que la seguridad social llegue a todos los ciudadanos, de modo que en el caso de las personas que no hayan ahorrado lo suficiente para vivir una vejez digna con una pensión mínima, se provean los recursos que la hagan posible (Ruiz, 2005).

\section{Recomendaciones para darle solvencia a la Seguridad Social}

Tras haber descrito la problemática de los sistemas de seguridad social, se presentanalgunasrecomendaciones con la finalidad de darle viabilidad a los mismos, ya que sería muy penoso que se llegara en el futuro a presenciar lo que algunos autores pronostican: la desaparición de la jubilación.

En cuanto a la edad de jubilación, ésta debe ser una decisión personal de cada individuo y no debe fijarse arbitrariamente. Para esto se sugiere definir de manera adecuada la relación entre la edad y el salario de retiro, ya que un individuo que dure más años laborando merece un mayor salario de retiro, pues esto representa una ventaja financiera para su fondo jubilatorio. Un esquema apropiado es el que han adoptado varias universidades públicas en México, que las tasas de reemplazo se relacionen a la suma de edad y antigüedad del trabajador, recomendándose una tasa del $100 \%$ para una suma de edad y antigüedad no menor a 95 años. De manera similar, se sugiere a las empresas y organizaciones averiguar las causas por las cuales sus empleados toman la decisión de jubilarse, con el fin de idear estrategias tendientes a incentivar su permanencia en el empleo.

Para las aportaciones a las cuentas individuales de retiro, se propone un esquema donde aporten el patrón, el gobierno y el mismo empleado y que lo hagan en una cantidad tal, que garantice el mismo nivel de vida del trabajador cuando llegue al retiro. Además que se incluya la posibilidad de que si el empleado desea aumentar su ahorro hasta una cantidad máxima previamente establecida, tanto el patrón, como el 
gobierno, hagan aportaciones por cantidades iguales a la del trabajador.

Se recomienda que se regule el manejo de las cuentas de retiro de los mexicanos por las Administradoras de Fondos para el Retiro (AFORES), ya que los rendimientos que ofrecen son muy bajos comparados con las administradoras de otros países, pues las comisiones que cobran son excesivamente elevadas. Otra sugerencia es utilizar los fondos que se destinan para la vivienda como parte del portafolio de inversiones de retiro, con la finalidad de obtener mejores rendimientos y que tales fondos sean portables en caso que los trabajadores no los utilicen para hacerse de una casa, de modo que puedan aprovecharlos donde más les convenga.

Se recomienda establecer salarios reguladores de jubilación con topes máximos y mínimos. El límite máximo puede establecerse una cantidad equivalente a 30 salarios mínimos y el mínimo en 2 salarios mínimos. Otra sugerencia para los jubilados es la de formar uniones de personas retiradas, ya que juntas pueden realizar actividades grupales y presionar al gobierno por mejores servicios, como el de salud y otros.

Se recomienda a las empresas e instituciones buscar la forma de implementar la jubilación flexible, para lo cual deberán tener en cuenta los siguientes aspectos:

- Definir los objetivos a cumplir en la organización.

- Adecuar la normativa organizacional vigente.

\section{REFERENCIAS}

Aiman-Smith, L., Bergey, P., Cantwell, A.R., y Doran, M. (2006). The coming knowledge and capability shortage. Research-Technology Management, 49(4), 15-23. .

Anderson, K., Burkhauser, R., y Quinn, J. (1986). Do retirement dreams come true? The effect of unanticipated events on retirement plans. Industrial and Labor Relations Review, 39(4), 518-526.

Bould, S. (1980). Unemployment as a factor in early retirement decisions. American Journal of Economics and Sociology, 39(2), 123-136.

Burtless, G. (1986). Social security, unanticipated benefit increases and the timing of retirement. Review of Economic Studies, 53(176), 781-806.

Byham, W.C. (2008). Flexible phase - out. T\&D Magazine, 62(4), 34-37.

Cahill, K.E., Giandrea, M.D., y Quinn, J.F. (2006). Retirement patterns from Career employment. Gerontologist, 46(4), 514-523.

Cahill, K., Giandrea, M. y Quinn, J. (2011). Reentering the labor force after retirement. Monthly Labor Review, 134(6), 34-42.

Campos, J., López, A., Oropeza, M., Gómez, J.L., y Villa, A. (2001). Universidad Pública Mexicana: Seguridad Social y Problema Pensionario. Guadalajara: Ediciones de la Noche, Universidad de Guadalajara.

Cerezo, E., y Topa, G. (2008). La prejubilación en los miembros de alta dirección de las organizaciones: Un análisis cualitativo. Revista de Psicología del Trabajo y de las Organizaciones, 24(3), 389-416.

Christman, B. (2010). The next retirement hurdle: Why today's employees need more than a savings plan. Benefits Quarterly, 2 ${ }^{\text {nd }}$ Quarter, 30-35.

Coile, C., y Gruber, J. (2007). Future social security entitlements and the retirement decision. The Review of Economics and Statistics, 89(2), 234-246.

Consejo Nacional de Población. (2005). Proyecciones de la Población de México, 2005 - 2050. México: Impresores Profesionales.

Cortés, J.C. (2006). Política y seguridad social: Un reto permanente por la democracia. Revista Latinoamericana de Derecho Social, 2(EneroJunio), 379-385. 
Cremer, H., Lozachmeur, J.M., y Pestieau, P. (2004). Social security, retirement age and optimal income taxation. Journal of Public Economics, 88(11), 2259-2281.

Cutler, N. (2011). Working in retirement: The longevity perplexities continue. Journal of Financial Service Professionals, 65(4), 19-22.

Davies, J. (2005). When I'm sixty-five. People Management, Enero 13, 11(1), 17.

Duval, R. (2004). Retirement behaviour in OECD countries: Impact of old-age pension schemes and other social transfer programmes. OECD Economic Studies, 37, 7-50. Disponible en http://www.oecd. org/dataoecd/12/23/34561950.pdf .

Dwyer, D.S., y Mitchell, O. (1999). Health problems as determinants of retirement: Are self-rated measures endogenous? Journal of Health Economics, 18(2), 173-193.

Dychtwald, K., y Baxter, D. (2007). Capitalizing on the new mature workforce. Public Personnel Management, 36(4), 325-334.

Erickson, T.J. (2008). Reinvent your career, and get ready for the next 30 years. T\&D Magazine, 62(7), 37-39.

Fields, G.S., y Mitchell, O.S. (1984). Economic determinants of the optimal retirement age: An empirical investigation. Journal of Human Resources, 19(2), 245-262.

Filer, R., y Petri, P. (1988). A job-characteristic theory of retirement. Review of Economics \& Statistics, 70(1), 123-128.

Franklin, M.B. (2011). 5 steps to a secure retirement. Kiplinger's Personal Finance, 53-58.

Geber, B. (2000). Who will replace those vanishing execs. Training, 37(7), 48-56.

Hall, A., y Johnson, T.R. (1980). The determinants of planned retirement age. Industrial and Labor Relations Review, 33(2), 241-254.

Hayashi, C., Olkkonen, H., Sikken, B.J., y Yermo, J. (2010). Transforming pensions and health care in a rapidly ageing world: Opportunities and collaborative strategies. Pensions, 15(3), 161-174.

Holzmann, R. (2000). The world bank approach to pension reform. International Social Security Review, 53(1), 11-34.

Izar, J.M. (2006). Determinación de las variables que afectan al ahorro requerido para el retiro de los trabajadores. Revista Vértice Universitaria, 30(2),
27-34.

Izar, J.M. (2011). Los retos del retiro:Análisis del sistema de pensiones en México. México: Trillas.

Izar, J.M., Ynzunza, C.B. y Abreu, M. (2011). Implementación de la jubilación flexible en las Universidades Mexicanas con fondos pensionarios, una discusión teórica-práctica. Administración Contemporánea, Vol. III(15), 1-18.

Kinsella, K., y He, W. (2009). An aging world. International Population Reports, U.S. Government Printing Office, 1-196.

Laborsta Internet (2011). Economically active population, estimates and projections. Disponible en: http//laborsta.ilo.org/applv8/data/EAPEP/ eapep_E.html.

Levine, E.M. (2005). Monte Carlo simulation: Smart bet for baby boomers' retirement plans. CPA Journal, 75(9), 12-13.

Nelson, T. (2002). Ageism: Stereotypes and prejudice against older persons. Boston, MIT Press.

Noble, P., y Harper, E. (2010). Strategy and policy for phased retirement. Benefits Quarterly, $3^{\text {rd }}$ Quarter, 11-14.

Ruiz, Á.G. (2005). Los sistemas de pensiones de las universidades públicas en México. México: Porrúa.

Sargeant, M. (2004). Mandatory retirement age and age discrimination. Employee Relations, 26(2), 151-166.

Visco, I. (1999). Welfare systems, aging and work: An OCDE perspective, Conferencia en Brescia, Italia. New Welfare and Social Security in Europe, Sep 10-11, 1-29.

Vora, P.P., y McGinnis, J.D. (2000). The asset allocation decision in retirement: lessons from dollar-cost averaging. Financial Services Review, 9(1), 47-63.

Williamson, J., y McNamara, T. (May, 2003). The effect of unplanned events on retirement. Washington, USA, working paper, Fifth Annual Joint Conference, Retirement Research Consortium, 1-33. 